\title{
Quantitative evaluation of headache severity before and after endoscopic transsphenoidal surgery for pituitary adenoma
}

\author{
Amparo Wolf, MD, PhD, ${ }^{1}$ Sandy Goncalves, MSc, ${ }^{1}$ Fateme Salehi, MD, MSc, ${ }^{2}$ Jeff Bird, MD, ${ }^{2}$ \\ Paul Cooper, MD, ${ }^{3}$ Stan Van Uum, MD, PhD, ${ }^{4}$ Donald H. Lee, MBBCh, ${ }^{2}$ \\ Brian W. Rotenberg, MD, MPH, ${ }^{5}$ and Neil Duggal, MD, MSc ${ }^{1}$
}

'Division of Neurosurgery, ${ }^{2}$ Department of Medical Imaging, and ${ }^{3}$ Division of Neurology, London Health Sciences Centre, London; and ${ }^{4}$ Division of Endocrinology \& Metabolism and ${ }^{5}$ Department of Otolaryngology-Head \& Neck Surgery, St. Joseph's Hospital, London, Ontario, Canada

\begin{abstract}
OBJECTIVE The relationship between headaches, pituitary adenomas, and surgical treatment of pituitary adenomas remains unclear. The authors assessed the severity and predictors of self-reported headaches in patients referred for surgery of pituitary adenomas and evaluated the impact of endoscopic transsphenoidal surgery on headache severity and quality of life (QOL).

METHODS In this prospective study, 79 patients with pituitary adenomas underwent endoscopic transsphenoidal resection and completed the Headache Impact Test (HIT-6) and the 36-Item Short Form Health Survey (SF-36) QOL questionnaire preoperatively and at 6 weeks and 6 months postoperatively.

RESULTS Preoperatively, $49.4 \%$ of patients had mild headache severity, $13.9 \%$ had moderate severity, $13.9 \%$ had substantial severity, and $22.8 \%$ had intense severity. Younger age and hormone-producing tumors predisposed greater headache severity, while tumor volume, suprasellar extension, chiasmal compression, and cavernous sinus invasion of the pituitary tumors did not. Preoperative headache severity was found to be significantly associated with reduced scores across all SF-36 QOL dimensions and most significantly associated with mental health. By 6 months postoperatively, headache severity was reduced in a significant proportion of patients. Of the 40 patients with headaches causing an impact on daily living (moderate, substantial, or intense headache), $70 \%$ had improvement of at least 1 category on HIT- 6 by 6 months postoperatively, while headache worsened in $7.6 \%$ of patients. The best predictors of headache response to surgery included younger age, poor preoperative SF-36 mental health score, and hormone-producing microadenoma.
\end{abstract}

CONCLUSIONS The results of this study confirm that surgery can significantly improve headaches in patients with pituitary adenomas by 6 months postoperatively, particularly in younger patients whose preoperative QOL is impacted. A larger multicenter study is underway to evaluate the long-term effect of surgery on headaches in this patient group.

http://thejns.org/doi/abs/10.3171/2015.5.JNS1576

KEY WORDS pituitary adenoma; headache; endoscopic transsphenoidal surgery; quality of life; pituitary surgery

$\mathrm{P}$ ITUITARY tumors are relatively common, with reported prevalence rates ranging from $10 \%$ to $22 \%$ and accounting for $10 \%$ to $15 \%$ of all intracranial tumors. ${ }^{3,5,22}$ With the advent of neuroimaging, the incidental finding of pituitary adenoma has become much more frequent. Chronic headache is a common reason for undergoing neuroimaging. The incidence of headache in patients with pituitary adenomas has been reported to range between $33 \%$ and $72 \%,{ }^{1,6,23}$ The existence of any clear re- lationship between headaches, pituitary tumors, and surgical treatment of pituitary tumors remains to be elucidated.

Chronic headache in patients with pituitary tumors may arise from several putative mechanisms, including tumor volume and its relationship with the sellar structures, functional disturbance within the hypothalamopituitary axis, patient predisposition, and family history. ${ }^{8,9}$ Invasion of the cavernous sinus is a proposed etiology of headache given that the sinus contains the ophthalmic branch of

ABBREVIATIONS BP = bodily pain; $\mathrm{GH}=$ general health perceptions; HIT-6 = Headache Impact Test; $\mathrm{MH}=$ mental health; $\mathrm{PF}=$ physical function; $\mathrm{QOL}=$ quality of life; SF-36 = 36-Item Short Form Health Survey; RE = role-emotional; RP = role-physical; SF = social functioning; VT = vitality.

SUBMITTED January 11, 2015. ACCEPTED May 7, 2015.

INCLUDE WHEN CITING Published online October 23, 2015; DOI: 10.3171/2015.5.JNS1576. 
the trigeminal nerve and the internal carotid artery, the compression of which has the potential to generate pain. ${ }^{4}$ Gondim et al. found a direct association between the presence of headache and tumor size, optic chiasm compression, sellar destruction, and cavernous sinus invasion. ${ }^{9}$ Headaches can also occur in patients with hormone-producing adenomas, particularly growth hormone-secreting tumors and prolactinomas, and demonstrate improvement with medical endocrinological treatments. ${ }^{19}$ Few studies have investigated the impact of surgery on headaches in patients with pituitary adenomas. ${ }^{7,15}$

The purpose of the present study was to determine the severity of self-reported headaches in patients with pituitary masses before surgery and to prospectively evaluate the impact of purely endoscopic transsphenoidal surgery on headaches and QOL at 6 weeks and at 6 months postoperatively. The relationship between headache severity and factors, including hormone production, tumor volume, sellar extension, and cavernous sinus involvement, was examined along with potential predictors of headache response to surgery.

\section{Methods \\ Study Participants}

Our study prospectively collected data on 79 patients who underwent endoscopic transsphenoidal resection of pituitary adenomas between July 2009 and February 2014. This study was reviewed and approved by the Research Ethics Committee at London Health Sciences Centre. All patients gave written informed consent prior to inclusion in the study. At our institution, patients were managed by a multidisciplinary pituitary team consisting of a neurosurgeon, neurologist, otolaryngologist, neuroradiologist, neuroophthalmologist, and endocrinologist. All patients underwent endoscopic transsphenoidal surgery either for visual deterioration and/or endocrinological reasons. No patient underwent surgery for the treatment of headaches as the sole indication.

All patients underwent preoperative contrast-enhanced MRI of the head as well as postoperative MRI at 6 months to assess the extent of resection. Chiasm compression, cavernous sinus invasion, and suprasellar extension were determined on the basis of the radiological appearance and considered present or absent. Tumor volume was determined by the maximal diameters in 3 dimensions using the following formula: volume $=$ anteroposterior $\times$ craniocaudal $\times$ transverse $/ 2$. In addition, patients underwent a complete ophthalmological assessment and visual field analysis using the Humphrey Field Analyzer (Zeiss) before and after resection. Prior to surgery, all patients were followed up closely by the endocrinology and neurology teams and underwent routine testing of their pituitary hormones and target hormones.

Resection was performed jointly by an otolaryngologist and neurosurgeon. Generally, posterior nasal septectomy was performed to allow binasal access, and the face of the sphenoid and intersphenoidal septations were drilled away. The dura was incised, and the tumor was resected using standard endoscopic instruments. Any complex intraoperative CSF leak was repaired in a layered fashion, which consisted of a combination of Gelfoam; fat, muscle, and fascia grafting; dura matrix overlay; and a vascularized nasoseptal flap, followed by the placement of a lumbar drain postoperatively.

\section{Outcome Assessments}

The study participants were requested to complete the Headache Impact Test (HIT-6) and 36-Item Short Form Health Survey (SF-36) QOL questionnaires at 3 different time points: preoperatively and 6 weeks and 6 months postoperatively.

\section{HIT-6}

HIT-6 is a validated tool used to measure the impact that headaches have on daily functioning..$^{10,17}$ The final HIT-6 score is obtained from the simple summation of 6 items and ranges between 36 and 78 points, with larger scores reflecting a greater impact. The 4 headache impact severity categories are: little or no impact $(\leq 49)$, moderate impact (50-55), substantial impact (56-59), and intense impact (60-78).

\section{SF-36}

SF-36 assesses the general well-being of patients. This taxonomy has 3 levels: 1) items; 2) 8 scales that aggregate 2-10 items each; and 3) 2 summary measures that aggregate the scales. The 8 components are as follows: physical function (PF), role-physical (RP), bodily pain (BP), general health perceptions $(\mathrm{GH})$, vitality (VT), social functioning (SF), role-emotional (RE), and mental health (MH). The PF scale has been shown to be the best all-around measure of physical health, while the MH scale is the most valid measure of mental health in studies to date. ${ }^{16,24}$

\section{Statistical Analyses}

SPSS 20.0 (IBM Corp.) was used for all data analyses. The HIT-6 and SF-36 scores were expressed as the mean $\pm \mathrm{SD}$. The student paired t-test or ANOVA was used to compare mean HIT-6 and SF-36 preoperatively and 6 weeks and 6 months postoperatively. Pearson's correlation was used to assess the correlations between continuous variables. The chi-square test was used to compare categorical variables. The Wilcoxon sign-rank test was used to compare ordinal data across time points. Predictors of headache response to surgery were determined using logistic regression analysis. The $\alpha$ level was set to 5\%. All tests were 2-tailed analyses.

\section{Results \\ Study Patient Characteristics}

The baseline clinical characteristics of the 79 patients with pituitary adenomas are listed in Table 1 . The mean age \pm SD was $55.9 \pm 14.3$ years, and 36 patients were female. The mean tumor volume was $6.3 \pm 6.7 \mathrm{~cm}^{3}$. Seventy patients $(88.6 \%)$ had a sellar lesion with a maximum diameter greater than $10 \mathrm{~mm}$, and 9 patients $(11.4 \%)$ had microadenomas. Nineteen patients $(24 \%)$ had clinically relevant hormone-producing tumors, including tumors that resulted in acromegaly (growth hormone producing; $\mathrm{n}=10)$, produced thyroid-stimulating hormone $(\mathrm{n}=1)$, resulted in Cushing's disease (adrenocorticotropic hormone 
TABLE 1. Baseline characteristics of the study patients who underwent endoscopic transsphenoidal resection of pituitary adenomas

\begin{tabular}{cc}
\hline \multicolumn{1}{c}{ Characteristic } & Value $^{*}$ \\
\hline Mean age, yrs & $55.9 \pm 14.3$ \\
\hline Sex & $36(45.6)$ \\
\hline Female & $43(54.4)$ \\
\hline Male & $6.3 \pm 6.7$ \\
\hline Mean tumor size, $\mathrm{cm}^{3}$ & \\
\hline Tumor characteristic & $9(11.4)$ \\
\hline Microadenoma & $70(88.6)$ \\
\hline Macroadenoma & $66(83.5)$ \\
\hline Suprasellar extension & $54(68.4)$ \\
\hline Chiasm compression & $35(44.3)$ \\
\hline Cavernous sinus invasion & \\
\hline Tumor histology & $60(75.9)$ \\
\hline Null cell & $10(12.7)$ \\
\hline Somatotroph & $5(6.3)$ \\
\hline Corticotroph & $3(3.8)$ \\
\hline Prolactinoma & $1(1.3)$ \\
\hline Thyrotroph &
\end{tabular}

* Values are presented as the number of patients (\%) unless indicated otherwise. Mean values are presented as the mean \pm SD.

producing; $\mathrm{n}=5$ ), or resulted in prolactinoma (prolactin producing; $n=3$ ). Of the hormone-producing tumors, all but 2 patients with acromegaly achieved a biochemical cure postoperatively. Twenty-nine patients (36.7\%) had preoperative partial or complete anterior hypopituitarism. Based on the MRIs acquired preoperatively, $83.5 \%$ of patients had a tumor extending into the suprasellar space. Chiasmal compression was present in $68.4 \%$ of patients, and cavernous sinus invasion was reported in $44.3 \%$ of patients. Only 1 patient had a clinical history of pituitary apoplexy. Two patients had blood products reported on MRI.

Fifty-two percent of patients were not taking any regular analgesic medications. Forty percent of patients were reportedly taking ibuprofen or acetaminophen more than 3 days per week. Approximately $8 \%$ of patients were taking daily morphine or hydromorphone.

\section{Does Hormone Production Impact Headache Severity?}

The self-reported headache severity in the preoperative period, as categorized according to impact severity and measured by the HIT- 6 questionnaire, was as follows: mild in 39 (49.4\%) patients, moderate in $11(13.9 \%)$ patients, substantial in $11(13.9 \%)$ patients, and intense in 18 (22.8\%) patients (Fig. 1, "preoperative"). Younger age was correlated with increased preoperative headache severity (Pearson correlation: $r=-0.54, p<0.001$ ). Headache severity did not significantly differ between male and female patients. Hormone-producing tumors were significantly associated with increased severity of headaches compared with nonsecreting tumor $\left(\chi^{2}=11.6, p=0.009\right)$. Of the patients with hormone-producing tumors, 14 of 19 (73.6\%) patients had moderate, substantial, or intense headache se- verity compared with 26 of $60(43.3 \%)$ patients with nonsecreting adenomas. Hormone-producing tumors associated with increased severity of headache included 7 of 10 somatotrophs, 3 of 5 corticotrophs, 3 of 3 prolactinomas, and 1 of 1 thyrotroph.

\section{Does Tumor Volume, Suprasellar Extension, or Cavernous Sinus Invasion Impact Headache Severity?}

No statistically significant correlation was found between preoperative tumor volume and headache severity (Pearson correlation; $\mathrm{r}=-0.105 ; \mathrm{p}=0.419$ ). Furthermore, suprasellar extension, chiasmal compression, and cavernous sinus invasion of a pituitary tumor did not significantly predispose the patient to greater severity of headache $\left(\chi^{2}=\right.$ $4.5, \mathrm{p}=0.21 ; \chi^{2}=2.8, \mathrm{p}=0.41 ; \chi^{2}=3.6, \mathrm{p}=0.31$, respectively). In fact, patients with microadenomas $(<10 \mathrm{~mm})$ were more likely to have greater headache severity (moderate, substantial, or intense) that impacted their daily life $\left(\chi^{2}=5.9, p=0.029\right)$. The 2 patients with blood products on MRI reported moderate headaches preoperatively.

\section{What Is the Association Between Preoperative QOL and Headache Severity?}

Preoperative headache severity was found to be significantly associated with reduced scores across all SF-36 QOL dimensions, including $\mathrm{MH}$ (Pearson correlation, $\mathrm{r}=$ $-0.60 ; \mathrm{p}<0.001), \mathrm{BP}(\mathrm{r}=-0.58, \mathrm{p}<0.001), \mathrm{PF}(\mathrm{r}=-0.26$; $\mathrm{p}=0.02), \operatorname{RE}(\mathrm{r}=-0.52, \mathrm{p}<0.001), \mathrm{RP}(\mathrm{r}=-0.39, \mathrm{p}<$ $0.001), \mathrm{SF}(\mathrm{r}=-0.56, \mathrm{p}<0.001)$, VT $(\mathrm{r}=-0.31, \mathrm{p}=0.006)$, and $\mathrm{GH}(\mathrm{r}=-0.50 \mathrm{p}<0.001)$. Figure 2 depicts the scatterplot of the preoperative HIT- 6 scores and the 2 strongest inversely correlated factors: preoperative $\mathrm{MH}$ and $\mathrm{BP}$.

\section{What Is the Impact of Surgery on Headache Severity?}

A significant proportion of patients had decreased headache severity by 6 months postoperatively (Wilcoxon sign-rank test, $\mathrm{p}<0.001$ ) (Fig. 1). The percentage of patients with intense headache decreased from $22.8 \%$ in the preoperative period to $19.0 \%$ at 6 weeks and $7.6 \%$ at 6 months. The percentages of patients with substantial headaches decreased from $13.9 \%$ preoperatively to $10.1 \%$ and $5.1 \%$, respectively. Of the 40 patients with headaches causing impact on daily living (moderate, substantial, or intense headache), $70 \%$ patients had improvement in at least 1 category on HIT- 6 by 6 months postoperatively. Six patients $(7.6 \%)$ showed worsening of headache at 6 months by 1 grade (from Grade 1 to 2; or mild to moderate on the HIT-6 scale), and no patients had worsening by 2 grades.

Repeated measures ANOVA, which was used to compare the mean HIT-6 scores preoperatively and 6 weeks and 6 months postoperatively, shows a significant difference in the mean values across the 3 time points $(\mathrm{F}=3.2$, $p=0.042$ ). The post hoc Tukey's test showed a significant difference between the preoperative HIT- 6 (mean $=48.9$ \pm 11.8 ) and 6-month postoperative HIT-6 scores (mean $=$ $44.9 \pm 8.5)(\mathrm{p}=0.03)$, but not between preoperation and 6 weeks postoperation.

Similarly, when looking exclusively at the 60 patients with non-hormone-producing pituitary adenomas, there was a significant proportion of patients with decreased 


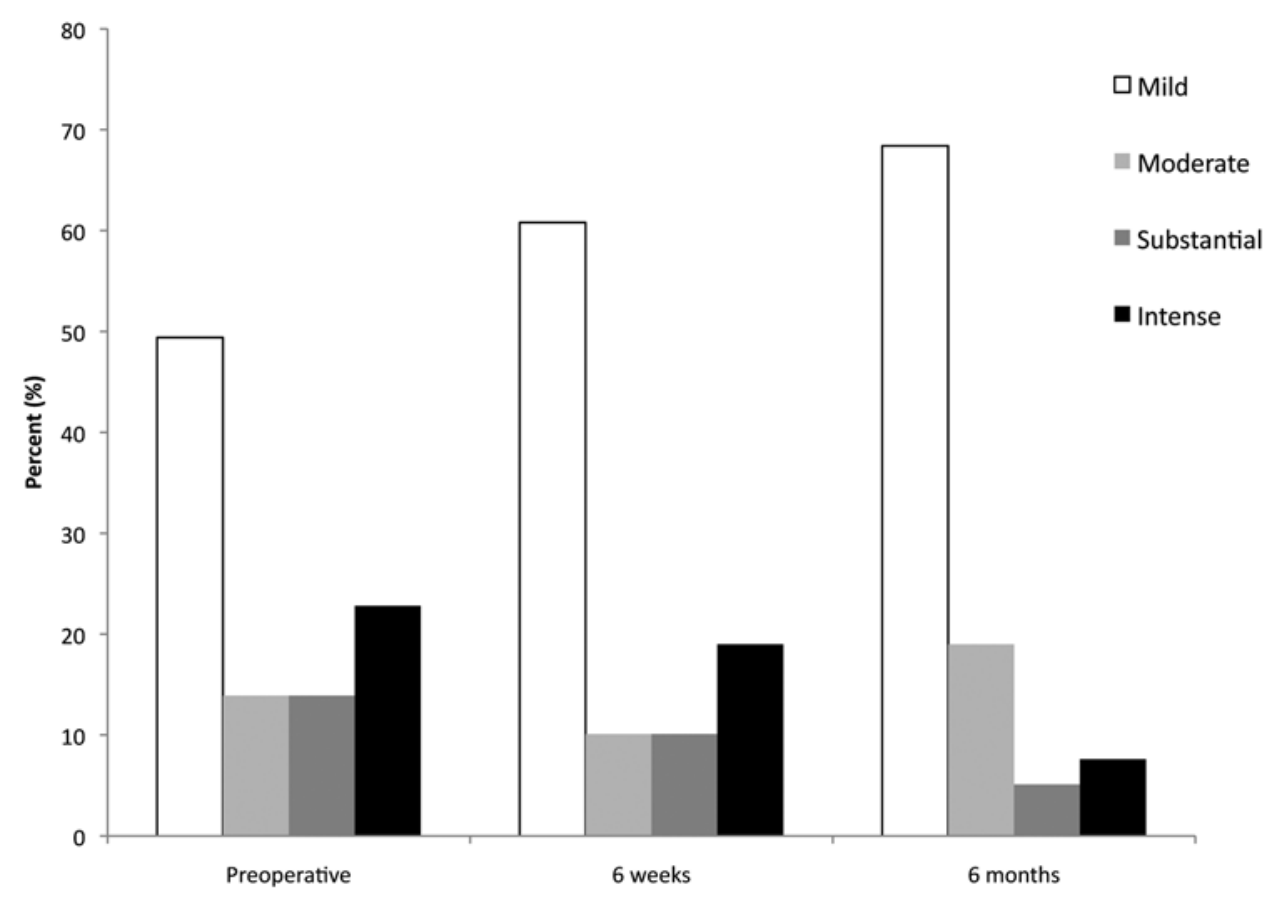

FIG. 1. Bar graph depicting the percentages of patients with mild, moderate, substantial, or intense headaches, as measured by the HIT-6 questionnaire, in the preoperative period and at 6 weeks and 6 months postoperatively.

headache severity by 6 months postoperatively (Wilcoxon sign-rank test, $\mathrm{p}=0.001$ ). As well, the mean HIT-6 scores were significantly reduced at 6 months postoperatively (paired t-test, $\mathrm{p}=0.009$ ).

Significant CSF leaks occurred in $12.7 \%$ of patients, which required the insertion of a lumbar drain. This patient population was more likely to have persistent headache at 6 months following surgery $\left(\chi^{2}=8.7, p=0.03\right)$.

\section{What Is the Impact of Surgery on QOL?}

A significant improvement in the mean SF-36 MH score was present when comparing the preoperative and 6 -week (paired t-test, $\mathrm{p}=0.012$ ) and 6-month postoperative scores $(p=0.023)$ (Table 2$)$. In addition, a significant improvement in the mean general health score was seen between the preoperative and 6 -week $(p<0.001)$ and 6 -month postoperative scores $(\mathrm{p}=0.012)$. The $\mathrm{BP}, \mathrm{SF}$, and $\mathrm{RP}$ scores showed significant improvement in comparison with the preoperative and 6-month postoperative scores ( $\mathrm{p}$ $=0.038, p=0.008, p=0.02$ respectively). No significant change was seen in the other SF-36 dimensions, including PF, RE, and VT. For patients with nonfunctioning macroadenomas, improvement in the $\mathrm{MH}$ scores was present at 6 weeks $(\mathrm{p}=0.037)$ and 6 months $(\mathrm{p}=0.050)$, as well in $\mathrm{GH}$ at 6 weeks $(\mathrm{p}=0.002)$.

\section{What Are the Predictors of Headache Response to Surgery?}

Age, functional microadenoma, preoperative pituitary insufficiency, and the SF-36 preoperative MH score were all associated with improvements in headache in response to surgery $\left(\chi^{2}=11.9, p=0.001 ; \chi^{2}=4.1, p=0.04 ; \chi^{2}=3.8\right.$, $\mathrm{p}=0.05 ; \chi^{2}=13.5, \mathrm{p}<0.001$, respectively), but not sex, suprasellar extension, chiasmal compression, or cavernous sinus invasion of pituitary tumors. Multiple logistic regression analyses were performed to determine the best predictors of response to surgery in all patients with pituitary adenomas using the factors that were significant on the univariate analysis. The logistic regression model was statistically significant $\left(\chi^{2}=17.9, \mathrm{p}<0.022\right)$. The model explained $36.2 \%$ (Nagelkerke $\mathrm{R}^{2}$ ) of the variance in response to surgery and correctly classified $68.0 \%$ of cases. The 2 best predictors are younger age and poor preoperative SF-36 MH score (Table 3).

\section{Discussion}

In this novel prospective study, we found that endoscopic pituitary surgery significantly improved headaches and QOL in patients with pituitary adenomas by 6 months postoperatively in comparison with preoperative baseline. Factors associated with increased headache severity preoperatively include hormone overproduction and younger age. Sex, tumor volume, suprasellar extension, chiasmal compression, and cavernous sinus invasion of pituitary tumors did not predispose patients to headaches. Younger patients with functional microadenomas and a significantly impacted MH QOL were more likely to improve with surgery.

The wide range in the reported incidences (33\%-72\%) and severity of headaches in patients with pituitary adenomas may be related to differences in pituitary tumor histology, size, and functional status. Factors previously reported to be associated with headache in patients with pituitary masses may be divided into either mechanical and/or biochemical causes., ${ }^{1,913,15}$ Our results are concordant with some previous reports showing that tumor vol- 

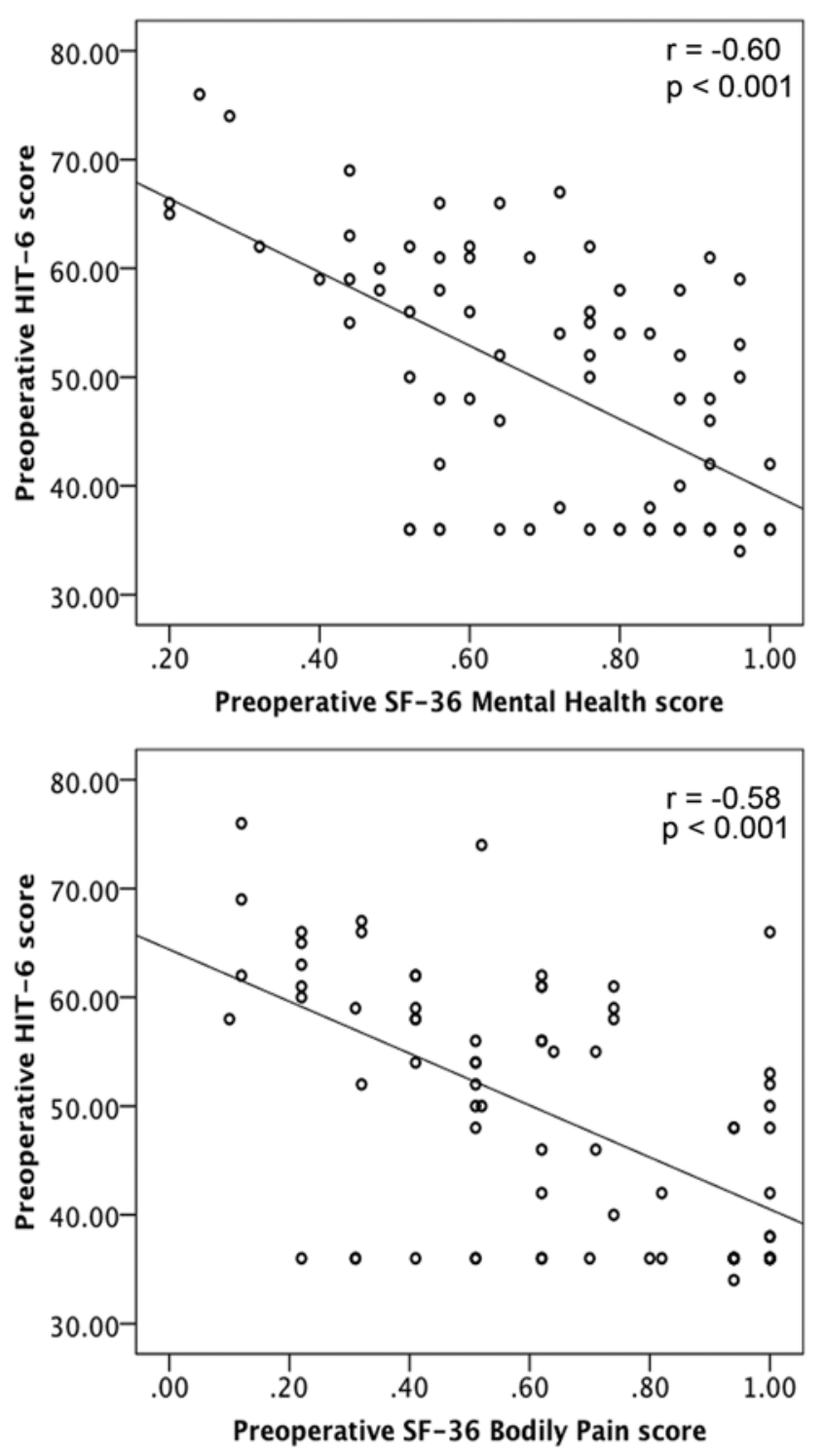

FIG. 2. Scatterplots showing a significant inverse correlation between preoperative HIT-6 scores and the SF-36 MH score (upper) (Pearson correlation: $r=-0.60, p<0.001)$ and the SF-36 BP score (lower) $(r=$ $-0.58, p<0.001)$.

ume and cavernous sinus invasion are not prerequisites for headache. ${ }^{1,13,15}$ It is unclear why disagreement exists in the literature regarding the impact of dural stretch and cavernous sinus invasion on the presence of headache. One possible explanation is that the phenotypic characteristics of pituitary tumor-related headaches and their outcome assessments differ across studies. This study focused on quantifying the severity of headaches that resulted in an impact in daily function.

Hormonal hypersecretion may be the underlying mechanism for some aspects of pituitary disease-related headaches. Growth hormone-secreting tumors and prolactinomas may be particularly nociceptive tumors and can potentially demonstrate improvement with endocrinological treatments. ${ }^{14,15,19}$ In our study, increased headache severity occurred in functioning tumors. Almost $74 \%$ of
TABLE 2. Summary of SF-36 scores for the study patients preoperatively and 6 weeks and 6 months postoperatively*

\begin{tabular}{cccl}
\hline $\begin{array}{c}\text { SF-36 } \\
\text { Domain }\end{array}$ & Preop & 6 Wks Postop & 6 Mos Postop \\
\hline MH & $0.71 \pm 0.21$ & $0.78 \pm 0.16 \dagger$ & $0.77 \pm 0.18 \dagger$ \\
\hline PF & $0.70 \pm 0.28$ & $0.67 \pm 0.26$ & $0.73 \pm 0.28$ \\
\hline BP & $0.64 \pm 0.29$ & $0.68 \pm 0.28$ & $0.71 \pm 0.26 \dagger$ \\
\hline RP & $0.58 \pm 0.44$ & $0.37 \pm 0.42$ & $0.71 \pm 0.39 \dagger$ \\
\hline RE & $0.71 \pm 0.40$ & $0.72 \pm-0.39$ & $0.79 \pm 0.34$ \\
\hline SF & $0.73 \pm 0.26$ & $0.72 \pm 0.26$ & $0.81 \pm 0.23 \dagger$ \\
\hline GH & $0.62 \pm 0.22$ & $0.70 \pm 0.20 \dagger$ & $0.68 \pm 0.22 \dagger$ \\
\hline VT & $0.50 \pm 0.34$ & $0.49 \pm 0.22$ & $0.54 \pm 0.25$ \\
\hline
\end{tabular}

* The results are presented as the mean \pm SD, with 0 representing a very low $\mathrm{QOL}$ for that item and 1 representing a very positive response. Changes over time were analyzed using the paired t-test.

$\dagger p<0.05$ vs the preoperative score.

patients with hormone-producing tumors in our study had headaches that impacted daily living (moderate, substantial, or intense) compared with $43 \%$ of patients with nonsecreting tumors. There were too few patients with each subtype of hormone-producing tumors to determine if a specific hormone was more prone to causing headaches, although all 3 patients with prolactinomas reported moderate headaches. In female patients with prolactinomas, there may be a higher frequency of headaches that were exacerbated by menstrual disturbances and related to prolactin hypersecretion. ${ }^{1}$ Inflammatory processes within the pituitary region, which result in enlargement of the pituitary due to edema and lymphocytic infiltration, is another putative mechanism of pituitary tumor-related headaches. To date, no specific biochemical factor has been directly associated with headache, including neuropeptide $\mathrm{Y}$, calcitonin gene-related peptide, substance $\mathrm{P}$, or vasoactive intestinal polypeptide..$^{11,12,18}$

Headache and hormonal disturbances can significantly worsen QOL in patients with pituitary adenomas. ${ }^{2}$ PereiraNeto et al. demonstrated that both the mental and physical dimensions of QOL, as measured by the RAND SF-36 measurement, were inversely correlated with headache intensities on the HIT-6 scale. ${ }^{20}$ Similarly, this study has found that preoperative headache severity is significantly associated with reduced scores across all SF-36 QOL dimensions, including mental health and general health. These results are intuitive in that patients with the greatest headache severity would suffer the greatest impact of the pain on their daily life and limitations in their individual capabilities.

Few studies have examined the impact of surgery on headaches and QOL in pituitary patients. Prior work by our group has shown that patient pain and discomfort are both decreased when undergoing endoscopic transsphenoidal resection in comparison with open microscopic surgery. ${ }^{21}$ For headaches, specifically, a single retrospective study with 41 patients found a significant improvement or resolution of headaches after endoscopic transsphenoidal resection in $90 \%$ of patients with nonfunctioning microadenomas and small Rathke cleft cysts. ${ }^{7}$ Another study 
TABLE 3. Multiple logistic regression analysis used to determine which factors predict the response of headache to endoscopic transsphenoidal resection in patients with pituitary masses

\begin{tabular}{llll}
\hline \multicolumn{1}{c}{ Predictor } & B Value & $\mathrm{p} \mathrm{Value}$ & \multicolumn{1}{c}{$95 \% \mathrm{Cl}$} \\
\hline Age & 0.952 & $0.026^{*}$ & $0.912-0.994$ \\
\hline Functional microadenoma & 3.01 & 0.205 & $0.547-16.5$ \\
\hline SF-36 MH (preop) & 0.02 & $0.005^{*}$ & $0.001-0.313$ \\
\hline Preop pituitary insufficiency & 0.561 & 0.137 & $0.171-1.833$ \\
\hline
\end{tabular}

${ }^{*} p<0.05$.

reported that $49 \%$ (27 of 54) of patients who underwent either transsphenoidal or transcranial resection of pituitary tumors demonstrated an improvement in headache following surgery, $36 \%$ experienced no change in symptoms, and $15 \%$ reported worsening of headache..$^{15}$ Our study is unique in that it prospectively evaluated the impact of purely endoscopic transsphenoidal surgery on headache severity at 6 weeks and 6 months postoperatively, without a confounding transcranial arm. By 6 months, $70 \%$ of patients who had been experiencing moderate, substantial, or severe headaches preoperatively reported reduced headache severity in at least 1 category on HIT-6. Improvement in headaches was seen at 6 weeks, but was statistically significant only at 6 months. Patients who had a significant postoperative CSF leak were less likely to show improvement of headache at 6 months. Surgery was also associated with significant improvement in several QOL dimensions, including $\mathrm{MH}$ and $\mathrm{GH}$. When analyzed independently, both nonfunctioning adenomas and hormone-producing adenomas benefited from surgery at 6 months. This suggests that there may be different underlying mechanisms at play that result in the improvement of headache severity after surgery.

The surgical indications in our study were 1) visual compromise secondary to chiasmal compression and/or 2) endocrinological abnormalities that were uncorrectable by medical therapy. Our results demonstrated that a patient's headaches significantly improve after endoscopic transsphenoidal surgery. However, the factors that determine who will benefit from intervention are unclear. Factors including tumor volume, suprasellar extension, and chiasmal compression did not correlate with headache severity or predict response to surgery. Predictors of headache response to surgery by at least 1 class on the HIT- 6 scale included patients with functional microadenomas and younger patients. Furthermore, patients with lower scores on SF-36's preoperative MH dimension were more likely to benefit from surgery.

There are several limitations to this study. A range of headache phenotypes can be found in patients with sellar masses, with migraine being the most frequent type. ${ }^{15}$ The headache characteristics and temporal profiles were not directly studied, but rather the impact of headaches on daily function. We did not have an adequate sample size of functional adenomas to comment on the impact of the subtype of hormone overproduction on headache severity. Follow-up times consisted of 6 weeks and 6 months and, as such, the long-term impact of surgery on headache remains unknown.

\section{Conclusions}

Headache is commonly associated with pituitary adenomas and may be an incapacitating symptom by impacting QOL and daily living. The goal of this study was not to determine whether headache alone should be considered an indication for surgical treatment of pituitary adenomas, but rather to describe the impact of surgery on the symptoms of headache and daily function in patients with pituitary tumors and visual dysfunction or endocrine abnormalities. We found that surgery can significantly improve headaches and QOL in patients with pituitary adenomas by 6 months. A larger prospective study is necessary to evaluate the long-term effects of surgery on headaches in patients with pituitary tumors and further develop the issue of headache as being a stand-alone indication for pituitary adenoma surgery.

\section{References}

1. Abe T, Matsumoto K, Kuwazawa J, Toyoda I, Sasaki K: Headache associated with pituitary adenomas. Headache 38:782-786, 1998

2. Baird A, Sullivan T, Zafar S, Rock J: Quality of life in patients with pituitary tumors: a preliminary study. Qual Manag Health Care 12:97-105, 2003

3. Buurman H, Saeger W: Subclinical adenomas in postmortem pituitaries: classification and correlations to clinical data. Eur J Endocrinol 154:753-758, 2006

4. Cottier JP, Destrieux C, Brunereau L, Bertrand P, Moreau L, Jan M, et al: Cavernous sinus invasion by pituitary adenoma: MR imaging. Radiology 215:463-469, 2000

5. Ezzat S, Asa SL, Couldwell WT, Barr CE, Dodge WE, Vance ML, et al: The prevalence of pituitary adenomas: a systematic review. Cancer 101:613-619, 2004

6. Ferrante E, Ferraroni M, Castrignanò T, Menicatti L, Anagni M, Reimondo G, et al: Non-functioning pituitary adenoma database: a useful resource to improve the clinical management of pituitary tumors. Eur J Endocrinol 155:823-829, 2006

7. Fleseriu M, Yedinak C, Campbell C, Delashaw JB: Significant headache improvement after transsphenoidal surgery in patients with small sellar lesions. J Neurosurg 110:354-358, 2009

8. Forsyth PA, Posner JB: Headaches in patients with brain tumors: a study of 111 patients. Neurology 43:1678-1683, 1993

9. Gondim JA, de Almeida JP, de Albuquerque LA, Schops M, Gomes E, Ferraz T: Headache associated with pituitary tumors. J Headache Pain 10:15-20, 2009

10. Kosinski M, Bayliss MS, Bjorner JB, Ware JE Jr, Garber $\mathrm{WH}$, Batenhorst A, et al: A six-item short-form survey for measuring headache impact: the HIT-6. Qual Life Res 12:963-974, 2003

11. Levy MJ, Classey JD, Maneesri S, Meeran K, Powell M, Goadsby PJ: The association between calcitonin gene-related peptide (CGRP), substance $P$ and headache in pituitary tumours. Pituitary 7:67-71, 2004

12. Levy MJ, Classey JD, Maneesri S, Meeran K, Powell M, Goadsby PJ: The relationship between neuropeptide Y expression and headache in pituitary tumours. Eur $\mathbf{J}$ Neurol 13:125-129, 2006

13. Levy MJ, Jäger HR, Powell M, Matharu MS, Meeran K, Goadsby PJ: Pituitary volume and headache: size is not everything. Arch Neurol 61:721-725, 2004

14. Levy MJ, Matharu MS, Goadsby PJ: Prolactinomas, dopamine agonists and headache: two case reports. Eur J Neurol 10:169-173, 2003

15. Levy MJ, Matharu MS, Meeran K, Powell M, Goadsby PJ: 
The clinical characteristics of headache in patients with pituitary tumours. Brain 128:1921-1930, 2005

16. McHorney CA, Ware JE Jr, Raczek AE: The MOS 36-Item Short-Form Health Survey (SF-36): II. Psychometric and clinical tests of validity in measuring physical and mental health constructs. Med Care 31:247-263, 1993

17. Nachit-Ouinekh F, Dartigues JF, Henry P, Becg JP, Chastan G, Lemaire N, et al: Use of the headache impact test (HIT-6) in general practice: relationship with quality of life and severity. Eur J Neurol 12:189-193, 2005

18. Nathoo S, Classey JD, Levy MJ, Meeran K, Powell M, Goadsby PJ: No relationship between vasoactive intestinal polypeptide expression and headache in pituitary tumours. Acta Neurol Scand 111:317-322, 2005

19. Pascual J, Freijanes J, Berciano J, Pesquera C: Analgesic effect of octreotide in headache associated with acromegaly is not mediated by opioid mechanisms. Case report. Pain 47:341-344, 1991

20. Pereira-Neto A, Borba AM, Mello PA, Naves LA, Araújo AS $\mathrm{Jr}$, Casulari LA: Mean intrasellar pressure, visual field, headache intensity and quality of life of patients with pituitary adenoma. Arq Neuropsiquiatr 68:350-354, 2010

21. Rotenberg B, Tam S, Ryu WH, Duggal N: Microscopic versus endoscopic pituitary surgery: a systematic review. Laryngoscope 120:1292-1297, 2010

22. Surawicz TS, McCarthy BJ, Kupelian V, Jukich PJ, Bruner JM, Davis FG: Descriptive epidemiology of primary brain and CNS tumors: results from the Central Brain Tumor Registry of the United States, 1990-1994. Neuro Oncol 1:14-25, 1999

23. Suwanwela N, Phanthumchinda K, Kaoropthum S: Headache in brain tumor: a cross-sectional study. Headache 34:435438, 1994

24. Ware JE, Gandek B: The SF-36 Health Survey: development and use in mental health research and the IQOLA project. Int J Ment Health 23:49-73, 1994

\section{Disclosure}

The authors report no conflict of interest concerning the materials or methods used in this study or the findings specified in this paper.

\section{Author Contributions}

Conception and design: Duggal, Cooper, Van Uum, Lee, Rotenberg. Acquisition of data: Duggal, Goncalves, Salehi, Bird, Cooper, Van Uum, Lee, Rotenberg. Analysis and interpretation of data: Duggal, Wolf, Goncalves, Salehi, Bird, Van Uum, Lee, Rotenberg. Drafting the article: Duggal, Wolf, Van Uum, Rotenberg. Critically revising the article: all authors. Reviewed submitted version of manuscript: all authors. Approved the final version of the manuscript on behalf of all authors: Duggal. Statistical analysis: Wolf, Goncalves. Administrative/technical/ material support: Cooper. Study supervision: Duggal, Cooper, Rotenberg.

\section{Correspondence}

Neil Duggal, London Health Sciences Centre, University Hospital, 339 Windermere Rd., London, Ontario N6A 5A5, Canada. email: neil.duggal@lhsc.on.ca. 